\title{
Cáncer sincrónico: neoplasias ginecológicas concurrentes de cuello, ovario y trompa. Caso clínico
}

\author{
Bárbara Castro Martín, Israel Ortega Sánchez, Javier de Santiago García, \\ Alicia Hernández Gutiérrez.
}

Servicio de Ginecología, Hospital Universitario la Paz, Madrid, España.

\section{RESUMEN}

Tumores ginecológicos primários dobles sincrónicos son relativamente frecuentes. Sin embargo los triple sincrónicos son extremadamente raros, más aún si uno de ellos es un tumor ginecológico extremadamente infrecuente, como es el cáncer de la trompa de Fallopio. Presentamos el caso de una mujer de 39 años con cánceres primarios sincrónicos del cuello uterino, ovario y trompa de Fallopio. No hay muchos casos descritos en la literatura, y casi todos ellos están relacionados con la mutación MSH2 (síndrome de Lynch) o BCRA. Es difícil diagnosticar un cáncer sincrónico preoperatoriamente y habitualmente son hallazgos después de cirugía profiláctica efectuada en pacientes con historia familiar de cáncer.

\section{PALABRAS CLAVE: Cáncer sincrónico, síndrome de Lynch}

\section{SUMMARY}

Double synchronous primary tumors of gynecological cancers are a relative common finding. However, triple synchronous primary gynecological cancers are an extremely rare event and even more if one of them it's the rarest gynecological tumor: the fallopian tube cancer. We present a 39- years old patient with synchronous cervical, fallopian tube and ovarian primary tumors. There are no many cases about similar reported in the literature and almost of all them have been related with gene mutations like MSH2 (Lynch syndrome) or BCRA. To diagnose synchronous cancers preoperatively is difficult and they 're usually unexpected findings after prophylactic surgery practiced in patients with family history of cancer.

\section{KEY WORDS: Synchronous cancer, Lynch syndrome}

\section{INTRODUCCIÓN}

La coexistencia de neoplasias primarias ó cáncer sincrónico es poco frecuente en el área ginecológica. Si bien la literatura recoge algunos ejemplos de doble neoplasia, la coexistencia de tres tumores primarios supone un hallazgo extraordinariamente raro (1). El diagnóstico en estos casos suele ser a posterior cuando realizada la cirugía, profiláctica ó con intención terapéutica hacia una neoplasia concreta, los hallazgos anatomopatológicos revelan la coexistencia de 2 ó más tumores primarios con distintas histologías.

En el caso que presentamos, destaca unido a lo particular de una triple neoplasia, el hecho de que además uno de los tumores sea un carcinoma tubárico, siendo éste el tumor ginecológico menos frecuente $(<1,5 \%)$. 


\section{Caso clínico}

Mujer de 39 años remitida a la Unidad de Ginecología Oncológica de nuestro hospital para valorar tratamiento, tras el diagnóstico anatomopatológico de adenocarcinoma de cuello uterino, en una muestra de biopsia cervical.

Sin antecedentes familiares o personales de interés. Refería la menarquia a los 11 años, ciclos regulares y dos embarazos previos a término, finalizados mediante cesárea. Sin control ginecológico en los últimos 9 años. La paciente consulta por irregularidades menstruales de un año de evolución, con genitorragia ininterrumpida durante los tres últimos meses.

A la exploración se objetiva un cérvix indurado con borramiento del labio posterior hasta fondo de saco vaginal, sin infiltrarlo. Se observa una erosion vera ligeramente excrecente con sangrado tanto al contacto como espontáneo. No se delimitan anejos y los parametrios impresionan libres. Se realiza colposcopia y biopsia con el resultado histológico de lesión altamente sugestiva de adenocarcinoma pobremente diferenciado. La ecografía transvaginal describe un cérvix hipertrófico con abundante vascularización e índices de resistencia superiores a 0,5 y una formación quística, tabicada, de contenido mixto de unos $8 \mathrm{~cm}$ en ovario izquierdo. El TAC abdomino-pélvico era concordante con la exploración anterior, calificando la formación anexial como hallazgo compatible con cáncer de ovario. Finalmente, tras obtener resultado negativo en el estudio de extensión, se programa la cirugía.

Se realiza laparotomía objetivándose "pelvis congelada" con anejos adheridos a cara posterior uterina y posible compromiso de parametrios. Se procede a la realización de histerectomía radical de Wertheim- Meigs con linfadenectomía pélvica y paraórtica, dejando un drenaje de Jackson en cavidad.

El estudio anatomopatológico posterior reveló los siguientes hallazgos: 1. Adenocarcinoma de cérvix bien diferenciado de tipo mucoso, sin invasión vascular, con afectación del límite cervical de uno de los parametrios y del borde de resección ectocervical en cara vaginal posterior. 2. Adenocarcinoma bien diferenciado intramucoso de trompa. 3. Tumor borderline de ovario, tipo mucoso. Endometrio con inflamación crónica, adenomiosis, endometriosis en serosa uterina y teratoma quístico maduro de ovario. Apéndice sin lesiones

El estudio ganglionar resultó negativo para los ganglios paraórticos (8 ganglios). Se analizaron 8 ganglios pélvicos derechos con metástasis en dos de ellos y 6 ganglios pélvicos izquierdos resultando sólo 2 positivos.

Durante el postoperatorio se trasfunden 2 concentrados de hematíes y pasadas las primeras 24 horas se observa un agravamiento de la paciente con inestabilidad hemodinámica y expulsión de material fecaloideo por el drenaje. Se confirma perforación intestinal que requiere colostomía de descarga, que fue realizada por el servicio de cirugía. Días después la paciente acusa molestias respiratorias; las pruebas de imagen confirman un derrame pleural derecho que responde favorablemente al tratamiento habitual, permitiendo el alta hospitalaria en el día 16 poscirugía. Una semana después se programa el inicio de radioterapia.

\section{DISCUSIÓN}

Durante los últimos años se ha observado un incremento en los casos documentados de cáncer sincrónico atribuyéndose esto a la mayor esperanza de vida de la población, a una mejora en los métodos diagnósticos ó incluso al avance de los tratamientos que impiden la diseminación de una primera neoplasia (2). De cualquier modo, aún así, el cáncer sincrónico supone un hallazgo inusual en la ginecología oncológica representando no más del $6 \%$ de los casos.

La asociación más frecuente en las series publicadas está formada por neoplasias de ovario y endometrio (2-3). Los cánceres de cuello, vagina y vulva, donde la presencia del virus del papiloma humano juega un papel importante, arrojan otro posible ejemplo de cáncer sincrónico a pesar de que en muchos de estos casos la detección es tardía, siendo prácticamente imposible diferenciarlos como tumores "independientes".

El enfoque cambia radicalmente con el tumor de trompa. Las neoplasias tubáricas suelen corresponder a diagnósticos histológicos posoperatorios en el seno de cirugías ginecológicas varias, especialmente en aquellas realizadas bajo sospecha de un proceso maligno tal y como ocurre en el caso que presentamos (4-5). Su sospecha clínica es difícil y debido a la escasa bibliografía publicada al respecto, no se ha conseguido establecer una relación ni con otras neoplasias acompañantes ni tampoco con algún factor de riesgo identificable, salvo la predisposición genética hallada en casos de mutaciones BCRA o MSH (síndrome de Lynch), esto último más discutido (7).

Se ha postulado la teoría de la ovulación incesante tratando de explicar el cáncer de ovario, así como el efecto que los niveles estrogénicos elevados podrían desempeñar sobre el endometrio y con ello una eventual sincronía entre ambos tumores. 
Recientemente se han retomado estudios en esta línea, sin poder arrojar aún resultados concluyentes en cuanto a sincronía. De cualquier modo dicha teoría no sería aplicable para el cáncer de cérvix diagnosticado de forma concurrente en nuestro caso (6).

Sí parece más claro el factor genético predisponente que suponen las mutaciones comentadas. En el caso del síndrome de Lynch, la alteración en los genes "mismatch repair" (MMR), trasmitida mediante herencia autosómica dominante, conlleva junto a la predisposición para cáncer colorrectal un elevado riesgo de desarrollar entre otras, neoplasias de ovario y endometrio. Se cree que entre un $40-60 \%$ de las pacientes con síndrome de Lynch podrán desarrollar a lo largo de su vida un cáncer de endometrio. En cuanto al cáncer de ovario, el riesgo vital se situaría en un $10-12 \%$ y la mayoría de autores, aunque no arrojan cifras debido a la escasez de casos, califican de "nada desdeñable" la posibilidad de aparición sincrónica de ambas neoplasias (7).

El tumor tubárico inicialmente no pertenece al grupo de entidades características de este síndrome. Sin embargo, se han encontrado en diferentes series publicadas, neoplasias tubáricas en pacientes sometidas a cirugías profilácticas en el contexto de un síndrome de Lynch y ello ha abierto nuevas líneas de investigación al respecto (7).

Tumores primarios de trompa pueden encontrarse en cambio hasta en un $6 \%$ de los casos, en mujeres sometidas a salpingo-oforectomía bilateral profiláctica tras diagnóstico genético de una mutación BCRA1/BCRA2 pudiendo en estas pacientes protagonizar diferentes asociaciones sincrónicas. De todos modos, aunque la paciente que presentamos no aportaba estudio genético, no parece que las mutaciones previamente comentadas pudieran explicar los hallazgos, dada la anamnesis y la ausencia de antecedentes oncológicos familiares de interés. Pudiéramos pensar en este punto, en mutaciones de novo o alteraciones génicas todavía por descubrir (8).

Se ha destacado también respecto al cáncer sincrónico que en la mayoría de las series publicadas las pacientes afectas son jóvenes o de mediana edad (39 años, en nuestro caso), en cualquier caso premenopáusicas en contra de lo característico para la mayoría de neoplasias ginecológicas aisladas. Igualmente, se habla de mayor porcentaje de nuliparidad y obesidad si bien esto no se produce en el caso que presentamos (6).

Respecto a los casos de enfermedad metastásica, parece que los procesos sincrónicos, presentan mejor pronóstico con tasas de supervivencia libre de enfermedad y supervivencia global claramente superiores. La mayoría de los trabajos coinciden en argumentar el hallazgo de neoplasias en estadios más precoces y por lo general bien diferenciadas $(9,10)$. En nuestro caso, sí se cumple que tanto el tumor de cérvix como el tubárico son bien diferenciados, así como que este último y el ovárico corresponden a un estadio inicial pero en esta paciente, el tumor de cérvix ya presenta extensión ganglionar bilateral.

En un futuro los estudios moleculares podrían ser muy útiles para un diagnóstico más fiable, así como para la correcta filiación e incluso prevención de las neoplasias sincrónicas.

\section{BIBLIOGRAFÍA}

1. Phupong V, Khemapech N, Triratanachat S. Triple synchronous primary cervical, endometrial and ovarian cancers with four histologic patterns. Arch Gynecol Obstet 2007;276:655-8.

2. Cantu de Leon D, Perez Montiel D, Tabarez A, Martinez RM, Cetina L. Serous adenocarcinoma of the fallopian tube, associated with verrucous carcinoma of the uterine cervix: a case report of synchronic rare gynecological tumors. World J Surg Oncol 2009;7:20.

3. Kehoe S, Powell JE. Prior, synchronous and secondary malignancies in women with ovarian cancer. Int $J$ Gynaecol Obstet 2001;73:265-7.

4. Mekni A, Doghri R, Bel Haj Salah M, Tounsi H, Chelly I, Khadija B, Slim H, Nidhameddine K, Chelly H, Moncef $Z$. Simultaneous squamous carcinoma involving the fallopian tube and the uterine cervix. Arch Gynecol Obstet 2008;278:157-9.

5. Ayas S, Karateke A, Aköz I, Yenidede I. Primary serous carcinoma of the fallopian tube with synchronous cervical epidermoid carcinoma in situ: a case report. Eur J Gynaecol Oncol 2007;28:501-2.

6. Caldarella A, Crocetti E, Taddei GL, Paci E. Coexisting endometrial and ovarian carcinomas: a retrospective clinicopathological study. Pathol Res Pract 2008;204:643-8.

7. Palma L, Marcus V, Gilbert L, Chong G, Foulkes WD. Synchronous occult cancers of the endometrium and fallopian tube in a $\mathrm{MSH} 2$ mutation carrier at time of prophylactic surgery. Gynecologic Oncology 2008;111:575-8.

8. Tamiolakis D, Venizelos I, Nikolaidou S, Lambropoulou M, Bolioti S, Papadopoulos N. Ovarian mucinous cystadenocarcinoma with mural nodule of anaplastic carcinoma and synchronous cervical squamous carcinoma. Cesk Patol 2005;41:66-70.

9. Oranratanaphan S, Manchana T, Sirisabya N. Clinicopathologic variables and survival comparison of patients with synchronous endometrial cancer with ovaryan metastasis. Asian Pac J Cancer Prev 2008

10. Saglam A, Bozdag G, Kuzey GM, Kuçukali T, Ayhan A. Four synchronous female genital malignancies: the ovary, cervix, endometrium and fallopian tube. Arch Gynecol Obstet 2008;277:557-62. 